\title{
Postoperative Pain Perception and Patient's Satisfaction After Mandibular Third Molar Surgery by Primary Closure With Distal Wedge Surgery
}

\author{
Poompat Leechanavanichpan ${ }^{\mathrm{a}}$, Pirasut Rodanant ${ }^{\mathrm{a}, \mathrm{d}}$, Rachatawan Leelarungsun ${ }^{\mathrm{a}}$, \\ Natthamet Wongsirichat ${ }^{b, c, d}$
}

\begin{abstract}
Background: The aim of this study was to compare periodontal conditions of the distal aspect of mandibular second molar and patient's satisfaction between standard surgical technique and primary closure with or without distal wedge surgery used for the removal of mandibular third molar impaction (MTMI).
\end{abstract}

Methods: Twenty-four patients, aged 18 - 25 years, were invited to participate in this prospective, single-blinded, split-mouth randomized controlled study. Each participant owned similar bilateral impacted mandibular third molar. Periodontal parameters (i.e. probing depth (PD), gingival index (GI), plaque index (PI) and the distance from cusp tip to gingival margin (CT-GM)) were measured. The standard mandibular third molar surgery was performed on one side while for the other side the distal wedge surgery with or without osseous contouring was added to the protocol. Information about satisfaction was taken from questionnaire.

Results: At the site where distal wedge was performed, significant change in CT-GM occurred at all aspects and a significant PD reduction was observed at disto-buccal and mid-distal sites. Distance between cemento-enamel junction (CEJ) and gingival margin reduced significantly at all sites. No significant difference between two groups was found in GI, PI and patient's satisfaction.

Conclusions: Incorporating distal wedge surgery into MTMI removal protocol does improve periodontal health of adjacent second molar

Manuscript submitted April 17, 2019, accepted May 4, 2019

aDepartment of Advanced General Dentistry, Faculty of Dentistry, Mahidol University, Bangkok 10400, Thailand

${ }^{b}$ Department of Oral and Maxillofacial Surgery, Faculty of Dentistry, Mahidol University, Bangkok 10400, Thailand

${ }^{c}$ Walailak University International College of Dentistry, Phaya Thai District, Bangkok 10400, Thailand

${ }^{\mathrm{d} C}$ Corresponding Author: Pirasut Rodanant, Department of Advanced General Dentistry, Faculty of Dentistry, Mahidol University, 6 Yothi Street, Rajathevee, Bangkok 10400, Thailand. Email: pirasut.rod@mahidol.ac.th; Natthamet Wongsirichat, Department of Oral Maxillofacial Surgery, Faculty of Dentistry, Mahidol University, 6 Yothi Street, Rajathevee, Bangkok 10400, Thailand. Email: natthamet.won@mahidol.ac.th

doi: https://doi.org/10.14740/jocmr3841 and does not affect patient's satisfaction.

Keywords: Distal wedge surgery; Mandibular third molar impaction; Patient's satisfaction; Periodontal health

\section{Introduction}

Mandibular third molar impaction (MTMI) is an undeniable morbidity in dentistry. Unavoidable pain from impaction does disturb patient's comfort and normal activities which might decline their quality of life. Thus, surgical removal of the impacted tooth is introduced and recognized as a common procedure in dentistry [1]. The benefit of the procedure seems to be empirical, mostly from alleviation of pain, where patient's and dentist's expectations are met. However, periodontal consequences, commonly gingival pocket known as false- or pseudo-pocket, do evidently occur [2]. Pseudo-pocket is a clinical appearance of gingiva when the gingival margin is located more coronal to the cemento-enamel junction (CEJ) than physiologic position [3]. Eventually, not only with the contribution to the retention of food debris and microorganisms but also with the impediment of cleansing capability, these pseudopockets usually lead to the inflammation of the periodontium [4]. Periodontitis eventually occurs, and the uncomfortable feelings usually bring patients back for treatment.

It seems like the current standard technique for MTMI removal was designed to facilitate tooth removal without considering soft tissue management around second molar. Accordingly, the establishment of gingival pocket becomes an expected but ignored problem. The aim of this study was the evaluation of periodontal conditions on the distal aspect of mandibular second molar and patient's quality of life focusing on postoperative pain and discomfort from the integration of periodontal surgery (distal wedge operation [5]) to the surgical removal of MTMI.

\section{Materials and Methods}

\section{Patient selection}

Patients attending advanced general dentistry clinic demand- 
Table 1. Patient's Inclusion and Exclusion Criteria Including Withdrawal Criteria

\begin{tabular}{lll}
\hline Inclusion criteria & Exclusion criteria & Withdrawal criteria \\
\hline Age between 18 - 25 years; having good systemic & Pregnant or lactating mother; having serious medical & Patients could leave \\
health; no smoking; having symmetrically positioned & conditions such as hypertension; cardiovascular problems, & the study at any time \\
MTMI on both sides; not allergic to 4\% articaine with & renal and/or liver failure, or smoking habit; inability to & depending on their own \\
1:100,000 adrenaline; understanding and carrying out & attend the periodic examinations; taking any medication & decisions and will \\
the instructions given by the investigators; having & during the previous 5 days prior to the surgery that would & \\
given written informed consent for the study & alter their pain perception (e.g. analgesics, antidepressants)
\end{tabular}

ing for removal of MTMI were recruited in this prospective, single-blinded, randomized clinical trial using a split-mouth design study. Intraoral photographs and panoramic radiographs were taken. Further inclusion and exclusion criteria were shown in Table $1[6,7]$. The research protocol and informed consent were reviewed and approved by Mahidol University Institutional Review Board (COA.No.MU-DT/PY-IRB 2017/021.2303). The study was performed at the Advanced General Dentistry Clinic, Faculty of Dentistry, Mahidol University, Bangkok, Thailand.

The patients who approved to participate signed a consent form, ethics approval and the Helsinki Declaration. All patients were included in the interventions after receiving all the necessary instructions. In our research, the ethics statement has been conducted in full accordance with the World Medical Association Declaration of Helsinki. Every author of this study also has ORCID iD.

\section{Periodontal parameter measurements}

Gingival index (GI) [8], plaque index (PI) [9], and the distance from cusp tip to gingival margin (CT-GM) at the distal aspect of adjacent second molar of each participant were measured on the day of surgery and 3 months post-operation (Fig. 1). Probing depth (PD) and distance from cemento-enamel junction to gingival margin (CEJ-GM) were measured at 3 months post-operation.

\section{Surgical procedures}

The impacted mandibular third molars of each participant were randomly allocated into two groups: control group and study group. Standard surgical technique [10] was performed at control sites while the study sites underwent standard surgical technique combined with distal wedge operation with or without osseous surgery (Fig. 2). The washout period was 3 weeks before the second operation.

All surgical procedures were performed under local anesthesia (4\% articaine with 1:100,000 epinephrine) by the same operator. After tooth removal, the surgical field was rinsed with sterile $0.9 \%$ normal saline solution. The surgical wound was closed by interrupted suturing method with a 3/0 black braided silk. All participants received the same postoperative care instruction and medication. The medications include antibiotics (i.e. amoxicillin $1 \mathrm{gm}$ or clindamycin $300 \mathrm{mg}$ (if allergic to penicillin) orally twice daily for 5 days) and analgesics (i.e. paracetamol $1 \mathrm{gm}$ or ibuprofen $400 \mathrm{mg}$ orally twice daily). The operation time was recorded.

Each patient was seen for clinical evaluation and intraoral photography at 7 days (sutures were removed) and 3 months post-operation.

\section{Patient's satisfaction}

At 7 days and 3 months post-operation, all participants were

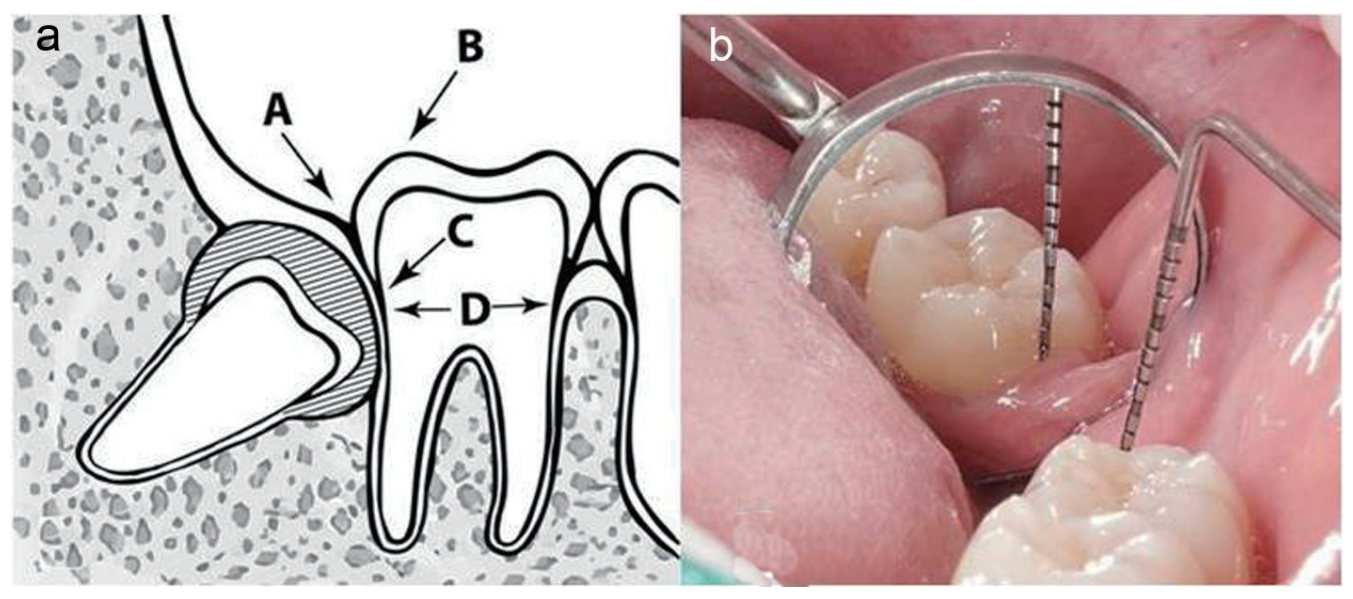

Figure 1. (a) Drawing picture of landmarks used for clinical measurements; gingival margin (A), cusp tip of mandibular second molar (B), cemento-enamel junction (C) and bottom of gingival pocket (D). (b) Intraoral photograph for the measurement of the distance from cusp tip to gingival margin using UNC15 probe. 


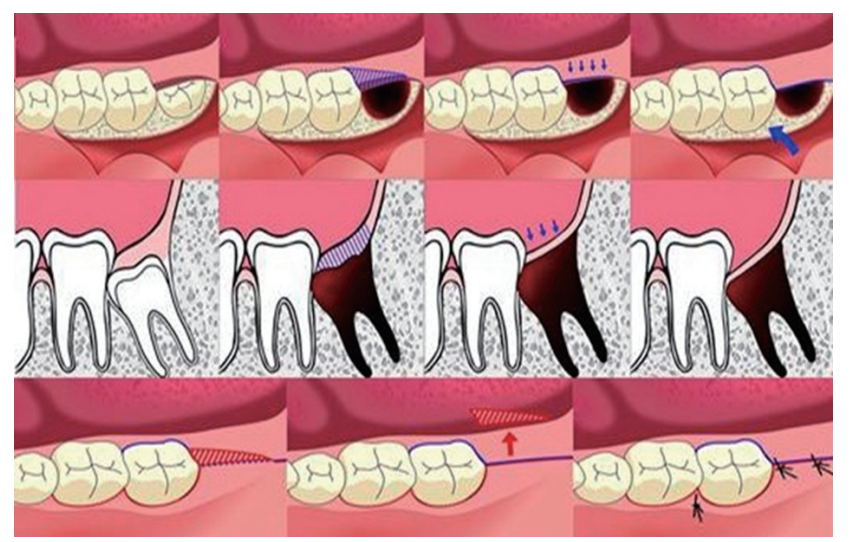

Figure 2. The study site undergoing surgical technique combined with distal wedge operation.

asked to complete a questionnaire consisted of questions concerning pain and discomfort. Patient's satisfaction was evaluated using visual analog scale (VAS) score [11, 12].

\section{Statistical analysis}

Statistical analysis was performed with SPSS $®$ (SPSS Inc, Chicago, IL, USA). Mean and SD of periodontal parameters and VAS score were calculated. For operation time we can test normality using Shapiro-Wilk test. Mean difference was assessed by Wilcoxon signed-rank test with significance level set at 0.05 .

\section{Results}

\section{Patient characteristics}

Twenty-four healthy patients (eight men and 16 women; mean age $20.27 \pm 2.26$ years, ranging $18-24$ years) were considered for inclusion in this study. A total of 48 MTMI were removed. All surgery was uneventful procedures except in two occasions (4.1\%) where postoperative complications occurred: an inferior alveolar nerve injury and a secondary infection. These two patients were excluded from the study; thus, a total of 22 patients were left for evaluation. Characteristics of MTMI were shown in Table 2.

For the operation time, it was found that the average time for control group was $45.68 \pm 20.18 \mathrm{~min}$ and for study group was $55.73 \pm 18.71 \mathrm{~min}$. We can study the dependent variable between groups for the "study group time" and "control group time". The operation time was normally distributed, because the significance value of the Shapiro-Wilk Test is greater than 0.05 . We also found that there is a significant difference between operation time of control and study groups at $\mathrm{P}<0.001$.

\section{Periodontal parameters}

The mean difference of CT-GM between baseline and 3 months post-operation and mean distance of CEJ-GM and PD at 3 months post-operation was shown in Table 3 .

The comparison between control and study groups found no significant differences in PI score and GI score at 3 months post-operation $(\mathrm{P}>0.05)$. Study group showed higher PI score $(2.00 \pm 0.82$ vs. $1.64 \pm 0.79)$ but lower GI score $(1.36 \pm 0.66$ vs. $1.73 \pm 0.77)$.

\section{Patient's satisfaction}

The participant's satisfaction was presented as mean \pm SD of VAS score in Table 4. There were no significant differences between study and control groups $(\mathrm{P}<0.05)$.

\section{Discussion}

This study demonstrated that the incorporation of periodontal surgical procedure into the standard surgical removal of mandibular third molar categorized as position B or C could benefit the reduction of gingival pocket and improvement of periodontal condition (Fig. 2) without affecting patient's postoperative satisfaction. Evidently, the presence of pre-surgical thick or bulk gingival tissue did associate with the occurrence of gingival pocket distal to the second molar after the MTMI removal with standard protocol [2]. Since the gingiva above MTMI categorized as position B or C is usually thick and its margin is high and approaching the occlusal surface of the second molar, these types of MTMI were selected in order to observe the effectiveness of distal wedge following MTMI removal. As for the healing potential, adolescents aged 18 - 25 years were recruited in order to eliminate the healing potential bias among participants [13].

After the study of the difficulty of operation, the results showed that there is a significant difference between operation time of control and study groups at $\mathrm{P}<0.001$, and that the difficulty of impaction removal played an important role in operation time and might account for postoperative pain. Difficulty seemed to be "bias" in this study which might af-

Table 2. Distribution of Class, Position and Angulation of MTMI

\begin{tabular}{lllllllll}
\hline \multirow{2}{*}{ Classification } & \multicolumn{3}{c}{ Position B } & & \multicolumn{3}{c}{ Position C } \\
\cline { 2 - 3 } Class I & Vertical & Mesioangular & Horizontal & & Vertical & & Mesioangular & Horizontal \\
\hline Class II & $6(13.6 \%)$ & $12(27.3 \%)$ & $4(9.1 \%)$ & & 0 & $10(22.7 \%)$ & 0 & $32(72.7 \%)$ \\
Total & 0 & $2(4.55 \%)$ & 0 & & 0 & $8(18.2 \%)$ & $2(4.55 \%)$ & $12(27.3 \%)$ \\
\hline
\end{tabular}


Table 3. The Mean Difference (Mean \pm SD, mm) of CT-GM Between Baseline and the Third Month, the Mean CEJ-GM and PD at the Third Month

\begin{tabular}{|c|c|c|c|}
\hline Group & Disto-buccal & Mid-distal & Disto-lingual \\
\hline \multicolumn{4}{|c|}{ CT-GM between baseline and the third month } \\
\hline Control & $1.27 \pm 1.12$ & $0.82 \pm 0.79$ & $0.72 \pm 0.94$ \\
\hline $\mathrm{P}$ value & $0.002 * *$ & $0.000 * * *$ & $0.001 * * *$ \\
\hline \multicolumn{4}{|c|}{ CEJ-GM at the third month } \\
\hline$P$ value & $0.02 *$ & $0.004 * *$ & $0.000 * * *$ \\
\hline \multicolumn{4}{|c|}{ PD at the third month } \\
\hline Control & $3.00 \pm 0.87$ & $3.50 \pm 0.67$ & $3.00 \pm 0.62$ \\
\hline Study & $2.59 \pm 0.59$ & $3.09 \pm 0.53$ & $2.91 \pm 0.68$ \\
\hline
\end{tabular}

${ }^{*} \mathrm{P}<0.05,{ }^{* *} \mathrm{P}<0.01$ and ${ }^{* *} \mathrm{P}<0.001$. CT-GM: from cusp tip to gingival margin; CEJ-GM: from cemento-enamel junction to gingival margin; PD: probing depth.

fect postoperative pain. In order to reduce the effect of "bias", the following strategies were used: 1) The study design: single blinded (participants did not know which side is included in the study or control group), and split mouth design (to make the comparison more precise); 2) Type of MTMI (both sides possessed similar angulation, position and class to keep the homogeneity of samples); and 3) For sampling bias by the study design: randomized controlled trial using systematic random to allocate study and control groups.

Postoperative complications, inferior alveolar nerve injury and secondary infection, did occur in two participants possessing deep MTMI position C. It was not unexpected since high occurrence rate of these complications was reported in deep MTMI removal [14]. However, the participants were well treated and the symptoms eventually disappeared.

This study confirmed that MTMI removal combined with distal wedge surgery could improve periodontal condition. A significant increase in mean CT-GM represented gingival pocket depth reduction. There was significant difference in mean PD at all sites except the disto-lingual site while there was no significant difference in mean PI and GI. This observation is in agreement with the result obtained by Montero and Mazzaglia [15]. They reported that shrinkage of retro-molar soft tissue after a surgical removal of MTMI resulted in the reduction of PD but GI and PI remained unchanged. In contrast, the study conducted by Grondahl and Lekholm [16] showed that soft tissue shrinkage after surgical removal of impacted mandibular third molar did promote plaque control. In this study, despite the mean PI in study group was greater than that in control group, lower mean GI was observed. We postulated that lower mean GI in the study group was the result of distal wedge surgery which eliminated gingival pockets thus facilitating patient's homecare. Nevertheless, disto-lingual site of mandibular second molar is one of the most difficult areas to clean where greater mean PI might be experienced which consequently affected PD [17]. This might explain the occurrence of insignificant difference of mean PD at disto-lingual site between study and control groups. Accordingly, better mean GI, PI and PD were expected if participant could perform an adequate plaque control [18].

Participants in the study and control groups rated their satisfaction by VAS score at the same level at each evaluation. At day 7 , participants in study group seemed to experience more discomfort since they reflected higher mean VAS score. This might be the result of the distal wedge surgery which extended operation time. Nevertheless, the true benefit of distal wedge surgery revealed at 3 months post-operation when the healing process of soft tissue completed. The lower average VAS score representing satisfactory factors including food impaction, irritation, overall symptoms and daily activities in the study group could explain that the surgical procedure anticipating the gingival contouring around the distal aspect of the adjacent second molar did improve the periodontal condition and patient's satisfaction in the MTMI removal.

The consideration of "quality of life" is an important matter in the decision making procedure rather than the measurement of the procedure's outcome. Negative consequences of any treatment should be thoroughly identified at the planning stage to avoid any occurrences of the predictable drawback. The provided treatment cannot be considered to improve patient's quality of life if the patient has to come back for correction of the consequence [19]. This study indicated that by foreseeing the possibility of gingival pocket formation, strategic planning to incorporate distal wedge surgery into the standard surgical MTMI removal does not only prevent further morbidity by avoiding the unnecessary dental treatment but also provide patient's satisfaction which eventually improves quality of life.

\section{Conclusions}

Incorporating distal wedge surgery with or without osseous contouring into MTMI removal protocol does improve peri- 
Table 4. The Mean VAS Score at the Seventh Day and Third Month (Mean \pm SD)

\begin{tabular}{|c|c|c|}
\hline Symptoms & The seventh day (VAS) & The third month (VAS) \\
\hline \multicolumn{3}{|l|}{ Pain } \\
\hline Control & $1.35 \pm 1.52$ & 0 \\
\hline Study & $1.76 \pm 1.56$ & 0 \\
\hline $\mathrm{P}$ value & 0.147 & 1.000 \\
\hline \multicolumn{3}{|l|}{ Swelling } \\
\hline Control & $1.11 \pm 1.37$ & $0.01 \pm 0.04$ \\
\hline$P$ value & 0.195 & 0.317 \\
\hline \multicolumn{3}{|l|}{ Bleeding } \\
\hline Control & $0.60 \pm 0.71$ & $0.01 \pm 0.02$ \\
\hline Study & $1.20 \pm 1.74$ & 0 \\
\hline$P$ value & 0.73 & 0.317 \\
\hline$P$ value & 0.643 & 0.317 \\
\hline \multicolumn{3}{|l|}{ Trismus } \\
\hline Control & $1.14 \pm 1.57$ & $0.01 \pm 0.04$ \\
\hline Study & $1.38 \pm 1.72$ & $0.01 \pm 0.04$ \\
\hline$P$ value & 0.195 & 1.000 \\
\hline \multicolumn{3}{|l|}{ Irritation } \\
\hline Control & $1.76 \pm 2.64$ & $0.05 \pm 0.22$ \\
\hline Study & $2.20 \pm 2.50$ & $0.01 \pm 0.04$ \\
\hline$P$ value & 0.295 & 0.317 \\
\hline \multicolumn{3}{|c|}{ Overall symptom } \\
\hline \multicolumn{3}{|l|}{ Chewing } \\
\hline Control & $1.78 \pm 1.79$ & $0.01 \pm 0.04$ \\
\hline Study & $1.67 \pm 1.86$ & 0 \\
\hline$P$ value & 0.554 & 0.317 \\
\hline \multicolumn{3}{|l|}{ Talking } \\
\hline Control & $1.09 \pm 1.23$ & $0.01 \pm 0.06$ \\
\hline Study & $0.93 \pm 1.08$ & $0.01 \pm 0.06$ \\
\hline$P$ value & 0.615 & 1.000 \\
\hline \multicolumn{3}{|c|}{ Smiling or laughing } \\
\hline Control & $1.19 \pm 1.63$ & $0.01 \pm 0.02$ \\
\hline Study & $1.16 \pm 1.82$ & $0.01 \pm 0.04$ \\
\hline$P$ value & 0.776 & 0.317 \\
\hline \multicolumn{3}{|c|}{ Daily activities } \\
\hline Control & $0.83 \pm 1.29$ & $0.01 \pm 0.02$ \\
\hline Study & $1.19 \pm 1.81$ & $0.01 \pm 0.04$ \\
\hline $\mathrm{P}$ value & 0.348 & 0.317 \\
\hline
\end{tabular}


odontal status of adjacent second molar and does not affect patient's satisfaction. However, adequate plaque control still plays an important role in postoperative healing and the reestablishment of physiologic periodontium.

\section{Acknowledgments}

The authors would like to acknowledge the staff, dental assistants, including our colleagues in the Department of Advanced General Dentistry and the Department of Oral and Maxillofacial Surgery, Faculty of Dentistry, Mahidol University.

\section{Financial Disclosure}

This research received no external funding.

\section{Conflict of Interest}

The authors declare no conflict of interest.

\section{Informed Consent}

The informed consent was obtained, and reviewed and approved by Mahidol University Institutional Review Board (COA.No.MU-DT/PY-IRB 2017/021.2303).

\section{Author Contributions}

PR contributed to the conceptualization; PL was involved in formal analysis and data curation; PR and NW contributed to original draft preparation and project administration; PR and RL were involved in investigation and validation; PR, PL and NW contributed to reviewing and editing the manuscript, visualization and supervision; PR, PL, RL and NW contributed to the methodology and resources of the manuscript.

\section{References}

1. Koerner KR. Manual of minor oral surgery for the general dentist. 1st ed.; Wiley-Blackwell: Iowa, USA, 2006; p. 49-80.

2. Kugelberg CF, Ahlstrom U, Ericson S, Hugoson A. Periodontal healing after impacted lower third molar surgery. A retrospective study. Int J Oral Surg. 1985;14(1):29-40.

3. Carranza FA, Newman MG, Takei HH, Klollevold PR. Carranza's Clinical Periodontology. 12th ed.; W.B. Saunders Co.: Missouri, USA, 2015; p. 576-582.
4. Goldman H, Schluger S, Fox L, Cohen D. Periodontal Therapy. 3rd ed.; CV Mosby Co.: St. Louis, USA, 1964; p. 440-448.

5. Robinson RE. The distal wedge operation. Periodontics. 1966;4(5):256-264.

6. Pell G, Gregory B. Impacted mandibular third molars: classification and modified techniques for removal. Dent Digest 1933;39:330-338.

7. Winter GB. Principles of oxodontia as applied to the impacted mandibular third molar. 1st ed.; American Medical Book Co.: St Louis, USA, 1926; p. 241-279.

8. Loe H, Silness J. Periodontal disease in pregnancy. I. Prevalence and severity. Acta Odontol Scand. 1963;21:533551.

9. Silness J, Loe H. Periodontal disease in pregnancy. II. Correlation between Oral Hygiene and Periodontal Condtion. Acta Odontol Scand. 1964;22:121-135.

10. Mehra P, D'Innocenzo R. Manual of minor oral surgery for the general dentist. 2nd ed.; John Wiley \& Sons, Inc.: New Jersey, USA, 2016; p. 55-84.

11. Shugars DA, Benson K, White RP, Jr., Simpson KN, Bader JD. Developing a measure of patient perceptions of short-term outcomes of third molar surgery. J Oral Maxillofac Surg. 1996;54(12):1402-1408.

12. Rodanant $P$, Wattanajitseree K, Shrestha B, Wongsirichat N. Pain and quality of life related to suture removal after 3 or 7 days at the extraction sites of impacted lower third molars. J Dent Anesth Pain Med. 2016;16(2):131-136.

13. Kugelberg CF, Ahlstrom U, Ericson S, Hugoson A, Thilander H. The influence of anatomical, pathophysiological and other factors on periodontal healing after impacted lower third molar surgery. A multiple regression analysis. J Clin Periodontol. 1991;18(1):37-43.

14. Marciani RD. Complications of third molar surgery and their management. Atlas Oral Maxillofac Surg Clin North Am. 2012;20(2):233-251.

15. Montero J, Mazzaglia G. Effect of removing an impacted mandibular third molar on the periodontal status of the mandibular second molar. J Oral Maxillofac Surg. 2011;69(11):2691-2697.

16. Grondahl HG, Lekholm U. Influence of mandibular third molars on related supporting tissues. Int J Oral Surg. 1973;2(4):137-142.

17. Leung WK, Corbet EF, Kan KW, Lo EC, Liu JK. A regimen of systematic periodontal care after removal of impacted mandibular third molars manages periodontal pockets associated with the mandibular second molars. J Clin Periodontol. 2005;32(7):725-731.

18. Kan KW, Liu JK, Lo EC, Corbet EF, Leung WK. Residual periodontal defects distal to the mandibular second molar 6-36 months after impacted third molar extraction. J Clin Periodontol. 2002;29(11):1004-1011.

19. Poradzisz M, Florczak KL. Quality of life: input or outcome? Nurs Sci Q. 2013;26(2):116-120. 